\title{
EFICACIA DEL HIPOCLORITO DE SODIO AL 0,05\% EN LA INFLAMACIÓN GINGIVAL
}

\author{
EFECTIVENESS OF 0,05\% SODIUM HYPOCHLORITE RINSE AGAINST GINGIVAL \\ INFLAMMATION
}

\author{
Carolina Encalada Abad1,2,a, José Rafael Carhuancho-Aguilar², Ebingen Villavicencio-Caparó1
}

\begin{abstract}
RESUMEN
Objetivo: Comparar la efectividad de un enjuague bucal en base a hipoclorito de sodio al 0,05\% y clorhexidina al $0,12 \%$ en la disminución de la gingivitis. Material y método: estudio comparativo de grupos paralelos, aleatorizado doble ciego en 38 jóvenes militares del Ejército Ecuatoriano. Se emparejó a los participantes de acuerdo con sus métodos de higiene y se administró los enjuagues en dos grupos, posteriormente a una profilaxis profesional. Los enjuagues se realizaron dos veces al día por 30 segundos, con soluciones de $15 \mathrm{ml}$ de Clorhexidina al $0,12 \%$ y $15 \mathrm{ml}$ de NaOCl al $0,05 \%$. Se tomaron valores de Índice de Placa Bacteriana (QHPI), Índice Gingival de Löe y Silness y porcentaje de sitios con sangrado al sondaje en los días cero y 21. Se evaluaron efectos adversos mediante un cuestionario y el registro de fotografías para ver pigmentaciones. Resultados: No hubo diferencias estadísticamente significativas entre grupos. El día 21, los índices de placa disminuyeron en los dos grupos. En el grupo CLX, los valores QHPI promedio disminuyeron 0,38; en el grupo $\mathrm{NaOCl}$ disminuyeron 0,12. El valor IG promedio en el grupo CLX disminuyó 0,42 y en el grupo $\mathrm{NaOCl}$ 0,38. El porcentaje de sitios con sangrado al sondaje, disminuyó 3,84\% promedio en el grupo CLX y $7,07 \%$ en el grupo NaOCl. Conclusiones: La clorhexidina sigue siendo el "Gold Standard" como agente antiplaca y antigingivitis, pero el $\mathrm{NaOCl}$ al $0,05 \%$ produce un efecto comparable, lo que por lo que podría ser un método eficaz para combatir la inflamación gingival y acumulación de placa bacteriana a un menor costo.
\end{abstract}

Palabras clave: Hipoclorito de Sodio; clorhexidina; placa dental; índice gingival; gingivitis; enjuague bucal. (Fuente: DeCS Bireme).

\section{ABSTRACT}

Objectives: To compare the effectiveness of a mouth rinse of sodium hypochlorite $0.05 \%$ and $0.12 \%$ chlorhexidine against supragingival plaque and gingivitis development. Materials and Methods: A comparative randomized, double blind parallel group trial was performed with 38 young men who form part of the Ecuadorian Military Service. Subjects were divided into two groups according to their hygiene methods and each group receives a type of blinded rinse, after a professional cleaning. The rinses were indicated twice a day with rinses of Sodium Hypochlorite administered to Group 1 and $0,12 \%$ Chlorhexidine administered to Group 2, using $15 \mathrm{ml}$ of the substances twice a day during 60 seconds for 21 days. Plaque index (QHPI), Löe and Silness Gingival Index, and Percentage of bleeding sites on probing were recorded on Day 0 and Day 21. Adverse effects were evaluated with questionnaires and pictures were taken in order to register any staining. Results: Values between groups showed no statistically significant differences. On day 21 , the plaque indexes decreased in both groups. The CLX group, the average QHPI values decreased 0.38, in the $\mathrm{NaOCI}$ group the average values decreased 0.12 . The average value in the CLX IG group decreased 0.42 and 0.38 in the $\mathrm{NaOCl}$ group. The percentage of sites that bled on probing decreased $3.84 \%$ in average in the CLX group and $7.07 \%$ in the $\mathrm{NaOCl}$ group. Conclusions: Clorhexidine has evidence of "Gold Standard Agent" in terms of antiplaque and antigingivitis, but mouth rinses with $0.05 \% \mathrm{NaOCl}$ were effective compared to chlorhexidine in terms of antimicrobial action, which could be an effective method to treat inflammation gingival and the accumulation of bacterial plaque at a lower cost.

Keywords: Sodium hypochlorite; chlorhexidine; dental plaque; periodontal index; periodontal treatment; gingivitis. (Fuente: MeSH NLM).

${ }^{1}$ Universidad de Cuenca, Ecuador.

${ }^{2}$ Universidad San Martín de Porres- Perú.

a Máster en Investigación Médica

\section{Correspondencia:}

Carolina Encalada Abad.

Correo electrónico: caroencalada119@icloud.com
Este es un artículo Open Access distribuido bajo la licencia Creative Commons Atribución-NoComercialCompartirlgual 4.0

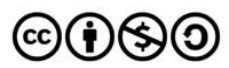

Citar como: Encalada C., Carhuancho JR., Villavicencio E. Eficacia del hipoclorito de sodio al 0,05\% en la inflamación gingival. KIRU. 2017;14(1):28 - 34. https://doi.org/10.24265/kiru.2017.v14n1.04 


\section{INTRODUCCIÓN}

Uno de los principales problemas de salud bucal es la acumulación de placa bacteriana supra gingival, que genera patologías como la gingivitis pudiendo progresar a pérdidas dentales graves por la agresividad de bacterias patógenas alojadas en la cavidad bucal. Con el paso del tiempo y los diferentes métodos de investigación, se ha logrado determinar que la eliminación de placa bacteriana reduce a su vez la gravedad de las enfermedades causadas por la misma ${ }^{(1)}$.

Los problemas inflamatorios periodontales son prevalentes en Latinoamérica: la gingivitis afecta al $34,7 \%$ de individuos jóvenes, con los valores más altos encontrados en Colombia (77\%) y Bolivia (73\%), y la menor prevalencia en México (23\%), mientras que en los demás países tuvo un rango entre el 31 y el $56 \%{ }^{(2)}$.

Esto se asocia con los factores de riesgo más comunes para los problemas gingivales $y$ periodontales, presentes en estas poblaciones, entre ellos la falta de higiene y un estado socioeconómico bajo, por lo que se ve necesario eliminarlos con medidas preventivas. Para el control de placa bacteriana se utiliza varias sustancias, siendo la Clorhexidina al $0,12 \%$ el enjuague Gold Standard. Tiene actividad bacteriostática a concentraciones bajas $(0,02 \%-0,06 \%)$, afectando el balance osmótico de las células de las bacterias, mientras que a concentraciones más altas (> $0,12 \%$ ) actúa como bactericida ${ }^{(3)}$. Sin embargo, el costo elevado de esta sustancia es uno de los principales limitantes. Por su parte el mecanismo de acción del hipoclorito de sodio consiste en reaccionar con las proteínas, ácidos nucleicos y lípidos, e inactivar las enzimas que son esenciales para la liberación de energía de los microorganismos ${ }^{(4)}$.

A pesar de que la erradicación total de la placa dental es imposible, la eliminación de placa bacteriana produce una mejor salud gingival. Estudios remarcan el hecho de que la placa bacteriana, y su permanencia en contacto con el margen gingival, producirán gingivitis. Los dientes que se encuentran libres de placa bacteriana presentan una flora escasa, colonizada casi exclusivamente por cocos y bacilos gram-positivos. Conforme se acumula la placa a lo largo del tiempo existen cambios en la composición de la misma, considerando de mayor gravedad la calidad que la cantidad $^{(5)}$.

Al determinar la naturaleza bacteriana de las enfermedades periodontales, la literatura reporta tratamientos quirúrgicos, no quirúrgicos, sistémicos y locales para tratar esta condición y el sector industrial, por su parte, presenta productos rentables que suponen tener efectos a largo plazo(6).

El manejo de la microbiota bucal con drogas antibióticas y agentes antisépticos, adicional a la eliminación mecánica, parece ser más eficaz que la terapia mecánica en sí. Estudios realizados previamente sugieren que los enjuagues con agentes antisépticos puede ser un método para controlar la inflamación periodontal y la acumulación de placa bacteriana, al aumentar el efecto local sobre los microorganismos que la constituyen ${ }^{(7)}$. Esto ha provocado mayor interés en la investigación de agentes antisépticos de bajo costo, concretamente el hipoclorito de sodio a bajas concentraciones, por su capacidad disolutiva de tejido orgánico ${ }^{(8)}$. Se realizó este estudio en vista de las precarias condiciones de salud bucal y alto índice de placa bacteriana y gingivitis en el Ecuador para que con bajos recursos se logre mejores índices de salud bucal y se contrarreste la gingivitis y acumulación de placa bacteriana. El objetivo del estudio fue comprobar la efectividad del hipoclorito de sodio en la reducción de placa bacteriana y gingivitis.

\section{MATERIALES Y MÉTODOS}

Se trabajó con 38 jóvenes en servicio militar de la Brigada de artillería $N^{\circ}$. 27 Portete, CuencaEcuador, con un mínimo de 20 dientes naturales en boca y encías sanas o periodontitis leve, con pérdida de inserción clínica menor o igual a $2 \mathrm{~mm}$. Fueron excluidos sujetos con periodontitis moderada o avanzada, dientes con caries que comprometieren más de un tercio de la corona, enfermedades sistémicas como diabetes, $\mathrm{VIH}$ o gingivitis o periodontitis úlceronecrotizante, tterapia sistémica inmunosupresiva, terapia antibiótica, antinflamatoria 0 anticoagulante, tomada un mes antes de la examinación inicial, pacientes bajo uso de medicamentos causantes de agrandamientos gingivales, flujo salival reducido, fumadores de más de 10 cigarrillos diarios, sujetos con alguna condición que requiera profilaxis antibiótica previa a un tratamiento odontológico invasivo y portadores de prótesis dentales o aparatología ortodóntica.

Se impartió una charla informativa de salud bucal y del estudio al personal militar; se realizó un examen bucal, profilaxis y destartraje. Se les explicó las condiciones del estudio y se les solicitó la aprobación del consentimiento informado. Los sujetos seleccionados comenzaron el experimento tres semanas después de la profilaxis realizada (día cero), pasaron por una valoración de gingivitis usando el índice gingival modificado(9), seguido de un sondaje periodontal, toma de fotografías y 
llenado de índice de placa, utilizando el índice de Quigley-Hein modificado por Turesky ${ }^{(10)}$, además de enseñanza y motivación sobre higiene oral con técnica de Bass modificada.

Los participantes fueron asignados a los grupos según tablas de números aleatorios a cargo de un colaborador. Los grupos fueron: G1, enjuague con clorhexidina (CLX 0,05\%) y $\mathrm{G} 2$, enjuague con hipoclorito de sodio ( $\mathrm{NaOCl}$ 0,05\%), asignación oculta para el investigador. El colaborador codificó diariamente las botellas correspondientes a las soluciones descritas y preparó la solución de $\mathrm{NaOCl}$ a diario a partir de una solución de $\mathrm{NaOCl}$ al $5 \%$ común o lavandina, y colocó $5 \mathrm{ml}$ de sustancia en $995 \mathrm{ml}$ de agua destilada de stock. La clorhexidina al $0,12 \%$ fue adquirida previamente preparada (Promeclin, Cuenca).

Las soluciones fueron asignadas y administradas a los pacientes a diario por parte del colaborador y supervisadas por la investigadora. Se aplicó el protocolo realizado por De Nardo y Cols, quienes experimentaron el hipoclorito de sodio en una población cautiva en Argentina(1). Desde el día cero, los sujetos fueron emparejados de acuerdo con el método de higiene bucal durante 21 días, y realizaron los enjuagues a diario durante 60 segundos con $15 \mathrm{ml}$ de las soluciones asignadas ${ }^{(1)}$ dos veces: en la mañana, luego del desayuno, y en la noche, luego de la merienda.

Cada semana se realizó un control fotográfico y consultas a los participantes del estudio para determinar posibles efectos adversos. Después del día 21, los sujetos recibieron una profilaxis y refuerzos de higiene oral junto con una aplicación de flúor tópico y operatoria dental en caso necesario.
Para valorar los cambios, se registró la información tomando registros de índice de placa e índice gingival y porcentaje de sitios con sangrado al sondaje, los días cero y 21 . Se realizó un control semanal con registros fotográficos para determinar posibles efectos adversos.

Para medir la acumulación de placa se utilizó el Índice de Quigley Hein modificado, escala del 0 al 5 (10). Para medir la salud gingival se utilizó el índice gingival (Löe- Silness), escala del 0 al $3^{(9)}$. El registro de sitios con sangrado al sondaje determinó el porcentaje de sitios que presentaron sangrado, indicativo de inflamación.

Se llenaron los valores en un formulario, se establecieron diferencias en cada superficie, al inicio y después de los enjuagues (días cero y 21). Se calculó la diferencia en cada paciente y se procedió al análisis de datos y pruebas estadísticas con el software SPSS v.19. Las diferencias de medias entre los grupos se analizaron con la prueba de Wilcoxon. No se pudo realizar pruebas de $\mathrm{Chi}^{2}$ por los valores qIG2 (índice de placa día 21) constantes. Se manejó un intervalo de confianza de $95 \%$ y valores $p: \leq 0,05$.

\section{RESULTADOS}

Treinta y ocho de los 42 sujetos terminaron el estudio. Fueron excluidos un sujeto por fractura mandibular, un portador de prótesis parcial superior, dos sujetos a terapia ortodóncica y dos bajo terapia antibiótica sistémica. Tres pacientes abandonaron el estudio previo al día cero por diagnóstico de varicela y uno con diagnóstico presuntivo de epilepsia. El promedio de edad fue de 19 años.

Tabla 1. Media de valores en día cero y día 21.

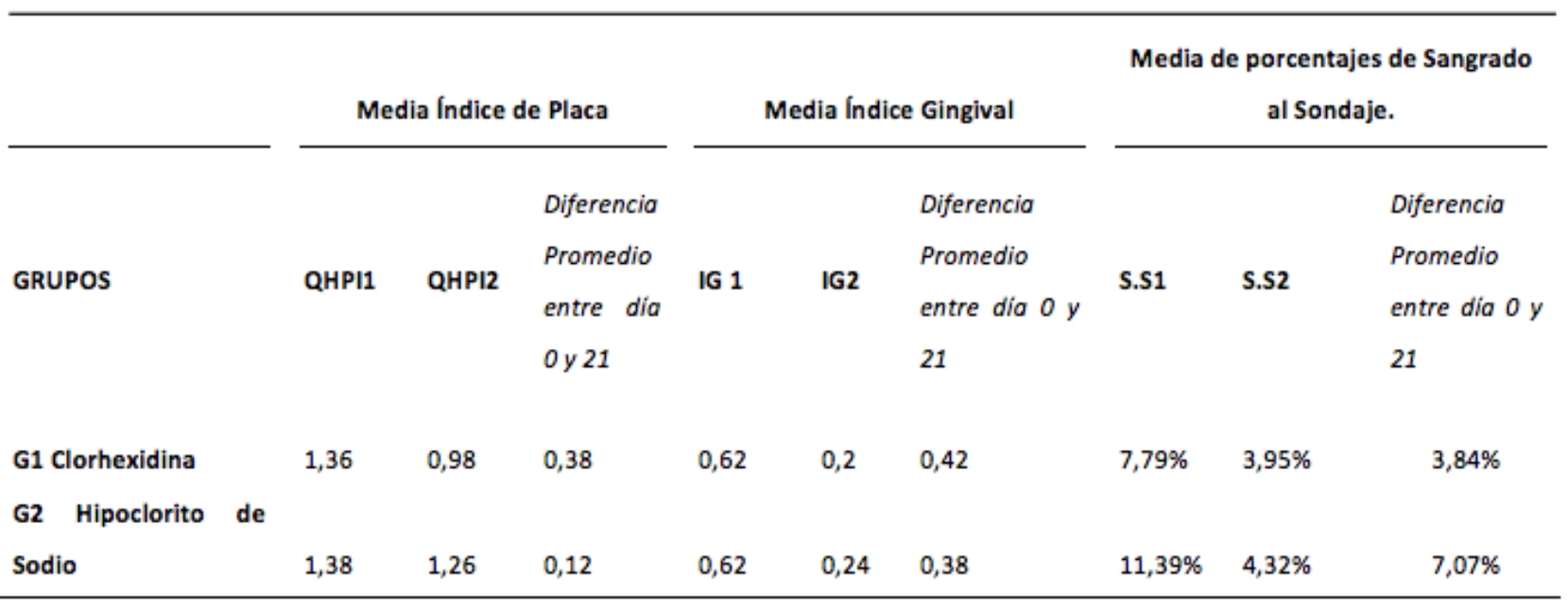

Elaborado por: Carolina Encalada A. 
La tabla 1 indica los valores clínicos tomados en el estudio. Los dos grupos presentaron valores similares al comenzar el estudio: el índice de placa bacteriana el día 21 disminuyó de 1,36 a 0,98 en el grupo $1(p=0,001)$ y de 1,38 a 1,26 en el grupo 2 $(p=0,334)$. La inflamación gingival el día 21 (IG2) disminuyó a 0,20 ( $p=0)$ en el $\mathrm{G} 1$, y a 0,24 ( $p=0,03)$ en el G2. El promedio de sitios con sangrado al sondaje, el día cero (SS1) en el G1 fue de 7,79\% y en el G2 11,39\%. El día 21 (S.S2) en el G1 mostró un promedio de 3,95\% ( $p=0,074)$ y el $\mathrm{G} 2$ de $4,32 \%$ $(p=0,011)$. Los pacientes de los dos grupos presentaron niveles de inflamación leve y moderado en el día cero. Se obtuvo únicamente valores de inflamación leve en el día 21. El 66,57\% del grupo clorhexidina presentó manchas extrínsecas. Del grupo hipoclorito, el $16,60 \%$ presentó manchas extrínsecas y el $100 \%$ refirió sabor fuerte del colutorio.

Tabla 2. Diferencia de medias.

\section{Grupo Clorhexidina}

\begin{tabular}{lccccccc} 
& QHPI2 - QHPI1 & IG2 - IG1 & S.S2 - S.S1 & & QHPI2 - QHPI1 & IG2 - IG1 & S.S2 - S.S1 \\
\hline $\mathbf{Z}$ & $-3,425^{c}$ & $-3,830^{c}$ & $-1,786^{c}$ & $\mathbf{Z}$ &,$- 966^{c}$ & $-2,167^{c}$ & $-2,533^{c}$ \\
$\begin{array}{l}\text { Sig. } \\
\begin{array}{l}\text { asintótica } \\
\text { (bilateral) }\end{array}\end{array}$ & 0.001 & 0 & 0.074 & $\begin{array}{l}\text { Sig. } \\
\text { asintótica } \\
\text { (bilateral) }\end{array}$ & 0.334 & 0.03 & 0.011 \\
\hline
\end{tabular}

Elaborado por Carolina Encalada A.

\section{DISCUSIÓN}

Con este estudio se pretendió, sobre todo, comprobar la existencia de una alternativa de higiene bucal para poblaciones cautivas con bajos recursos y situaciones precarias de salud bucal. De los resultados obtenidos en esta investigación, se puede establecer que al privar de higiene bucal por 21 días a los sujetos, siguiendo el modelo de "Gingivitis Experimental" de Loe Theilade y Jensen ${ }^{(5)}$, con administración de enjuagues bucales 2 veces al día, no aumenta el cúmulo de placa bacteriana ni empeora el índice gingival en el paciente.

La clorhexidina al $0,12 \%$, al ser utilizada como enjuague bucal "Gold Standard", con un 50,3\% $60,9 \%$ de reducción de placa bacteriana y un 30,5$42,5 \%$ de reducción de inflamación gingival ${ }^{(11)}$, produce un efecto previamente conocido, y según lo obtenido en esta investigación el enjuague con hipoclorito de sodio al $0,05 \%$ produce un efecto similar a la clorhexidina en cuanto a acción antigingivitis.

Al comparar los dos, según los valores promedio, el hipoclorito de sodio mostró una reducción de un 0,12 en la media de QHPI (CLXO. 38), una disminución de 0,38 en la media de IG (CLX 0,42) y el porcentaje de sitios con sangrado al sondaje se redujo de $11,39 \%$ a $8,33 \%(C L X$ de $7,79 \%$ a $3,95 \%)$.

De Nardo y cols $2012^{(1)}$, en un estudio realizado en 44 presos privados de higiene oral durante 21 días, con enjuagues de $\mathrm{NaOCl}$ diluido y agua destilada, muestran un aumento de 3,82 en valores de QHPI en el grupo de agua destilada y de 1,98 en el grupo de $\mathrm{NaOCl}$. En cuanto a índice gingival, el promedio en el grupo de agua destilada aumentó en 2,1 y en el grupo de $\mathrm{NaOCl}$ subió a 1 . El porcentaje de sangrado al sondaje aumentó a $93,1 \%$ en el grupo de agua destilada y a $56,7 \%$ en el grupo de $\mathrm{NaOCl}$. Las diferencias fueron estadísticamente significativas $(p=0,001)^{(1)}$. Al comparar con este experimento realizado, los valores de QHPI, IG y SS no muestran un aumento, sino una disminución. Los agentes utilizados en nuestro estudio, al comparar valores del día cero y día 21, disminuyen; lo que nos lleva a entender que los agentes utilizados combaten la acumulación de placa y gingivitis a pesar de la ausencia de otros métodos de higiene.

En los hallazgos encontrados en esta investigación se concuerda con el estudio de Lobene y cols ${ }^{(12)}$, quienes comprueban que el uso de instrumentos irrigantes y agentes antisépticos ( $\mathrm{H} 2 \mathrm{O} 2$ al 1\%, y $\mathrm{NaOCl} \quad$ al $0,5 \%)$ diluye 0 altera los productos tóxicos del metabolismo de la placa bacteriana causantes de gingivitis. Los dientes tratados con $\mathrm{NaOCl}$ demostraron una reducción de un $47 \%$ en placa bacteriana, al compararlo con $\mathrm{H} 2 \mathrm{O} 2$ y el grupo control. En este caso, los enjuagues bucales de clorhexidina e hipoclorito de sodio, sumados a la presión y movimiento generados en las buchadas, ayudan a la descomposición y desprendimiento de la placa bacteriana de las superficies dentales.

La clorhexidina al $0,12 \%$ en enjuague bucal tiene un efecto antiplaca y antigingivitis, caracterizada por 
su amplio espectro contra gram positivos y gram negativos, algunas levaduras y virus. Puede adherirse a la mucosa oral y a la película adquirida ampliando sus efectos, con la desventaja de producir pigmentación en uso a largo plazo ${ }^{(13)}$. El $\mathrm{NaOCl}$ es activo contra la mayoría de microorganismos, tiene una rápida acción antibacteriana, evita la adhesión de la placa a superficies sólidas, no presenta toxicidad relativa en la concentración utilizada, no pigmenta las piezas dentarias permanentemente, es de fácil acceso y de bajo costo ${ }^{(7)}$.

Si bien la clorhexidina al $0,12 \%$ ha sido utilizada en el tratamiento de gingivitis a lo largo del tiempo y el hipoclorito de sodio ha sido asociado a un mayor nivel de toxicidad al utilizarse como un enjuague; es importante aclarar el hecho de que la concentración en la que se utiliza ha sido previamente aceptada y recomendada por la $\mathrm{ADA}^{(14)}$.

Según lo planteado en esta investigación, es evidente la necesidad de mejorar la higiene bucal y salud gingival de los pacientes en condiciones cautivas. La gingivitis, resultante de la reacción de la encía al agente extraño, que en este caso es la placa bacteriana que se acumula en el surco gingival, se desarrolla según la respuesta del individuo y puede ser combatida con la terapia mecánica. En este contexto, los sujetos en servicio militar no presentan posibilidades económicas para instrumentos de salud bucal de alto costo, por lo que se plantea la alternativa sobre la utilización de un enjuague bucal fácilmente preparado y efectivo.

Jorgensen y cols $^{(7)}$ proponen una solución de enjuagues preparados por el paciente. En vez de utilizar enjuagues bucales de stock por su alto costo, adquirir clorhexidina genérica en concentraciones del $2 \%$ y diluirla en agua. En el caso del $\mathrm{NaOCl}$, se puede adquirir un frasco de lavandina ( $\mathrm{NaOCl} 5 \%$ ) y diluir una cucharita en dos vasos grandes de agua de 200-300mll ${ }^{(15)}$. La solución de cloro debe ser preparada a diario ya que este pierde su estabilidad en pocas horas.

Por lo antes citado, se puede decir que las expectativas planteadas para este estudio se cumplieron. Se quiso obtener resultados positivos con el enjuague bucal de $\mathrm{NaOCl}$ como agente anti placa y anti gingivitis, y estos son comparables a los valores logrados con el enjuague de Clorhexidina. Por lo tanto, si al uso de este enjuague de bajo costo y fácil acceso se le suma el efecto de terapia mecánica con una buena técnica de cepillado dental, los resultados serán más satisfactorios. Se considera por lo tanto una buena alternativa. Si se analiza la parte económica, se utilizaron $12 \mathrm{~L}$ de gluconato de clorhexidina, $105 \mathrm{ml}$ de hipoclorito de sodio y $12 \mathrm{~L}$ de agua destilada en los 21 días. El enjuague de clorhexidina resultó 2,11 veces más costoso que el enjuague de hipoclorito de sodio diluido, según los costos en el mercado local.

El $87 \%$ de pacientes comenzaron el día cero con inflamación leve y el 13\% con inflamación moderada que implica sangrado al sondaje. El día 21 todos los pacientes presentaron índices gingivales menores a 1, lo que indica inflamación leve o ausencia de inflamación.

Jorgen Slots y su grupo $(4,16,18-20)$ han estudiado a lo largo del tiempo alternativas de bajo costo para combatir enfermedades periodontales. Se refieren al hipoclorito de sodio como una sustancia segura por su alto espectro antimicrobiano. A pesar de su sabor incómodo, en bajas concentraciones es tolerable, seguro y no produce daño en las mucosas. Como efecto secundario puede producir manchas de color marrón, pero de fácil remoción en comparación con las producidas por la clorhexidina. Watts ${ }^{(21)}$, en su estudio sobre pigmentos dentales, explica que existe una variación en el grado de pigmentación de persona a persona, pudiendo ser causada por factores extrínsecos, intrínsecos o ambos. En el caso de la clorhexidina, se necesita mayor investigación para definir la razón exacta de la pigmentación. La mayor evidencia indica que probablemente resulta por los cationes absorbidos $^{(21)}$ que en vez de unirse a bacterias se unen a sustancias dietéticas ricas en taninos, produciéndose una pigmentación; así alimentos como el té, el vino tinto o el café potencian la pigmentación.

En este experimento, tres pacientes del grupo $\mathrm{NaOCl}$ presentaron pigmentaciones marrones en el cuello de las piezas dentarias, que como se mencionó pueden ser resultado de productos cromogénicos $^{(21)}$. En los demás individuos se pudo observar un color más blanco en la corona clínica de las piezas dentarias. En el grupo Clorhexidina, 7 sujetos presentaron pigmentaciones oscuras en la cara lingual de dientes antero inferiores. En cuanto al sabor del enjuague de $\mathrm{NaOCl}$, los pacientes reportaron un sabor fuerte pero tolerable sin efectos adversos.

Además del estudio de De Nardo, 2012(1), en el 2013, Galván M y cols ${ }^{(15)}$ realizaron un experimento que comparó $\mathrm{NaOCl}$ al $0,25 \%$ vs agua en pacientes con periodontitis leve y gingivitis, dos veces por semana durante 3 meses, sin detener otros métodos de higiene. Se tomaron valores al inicio, a las dos semanas y a los 3 meses. Observaron 94\% menos de placa bacteriana en el grupo de prueba y en el grupo control, 29\%. Encontraron aumentos del $94 \%$ y el $29 \%$ de las superficies vestibulares libres de placa, respectivamente. En cuanto a las superficies linguales, $195 \%$ menos con hipoclorito 
de sodio y $30 \%$ menos con agua. En cuanto a sangrado al sondaje, $42 \%$ de sitios sin sangrado al sondaje con $\mathrm{NaOCl}$ y en el grupo control, $29 \%$. Se demostraron así diferencias estadísticamente significativas.

Esta investigación, realizada en el personal militar, concuerda con los datos encontrados en estudios previos, pero no se han encontrado estudios que comparen directamente el enjuague de $\mathrm{NaOC}$ $0,05 \%$ vs CLX 0,12\%. La concentración utilizada de $\mathrm{NaOCl}(0,05 \%)$ está dentro de los límites de seguridad de la sustancia, y es recomendada para

Grupo 1. Clorhexidina

Día 0

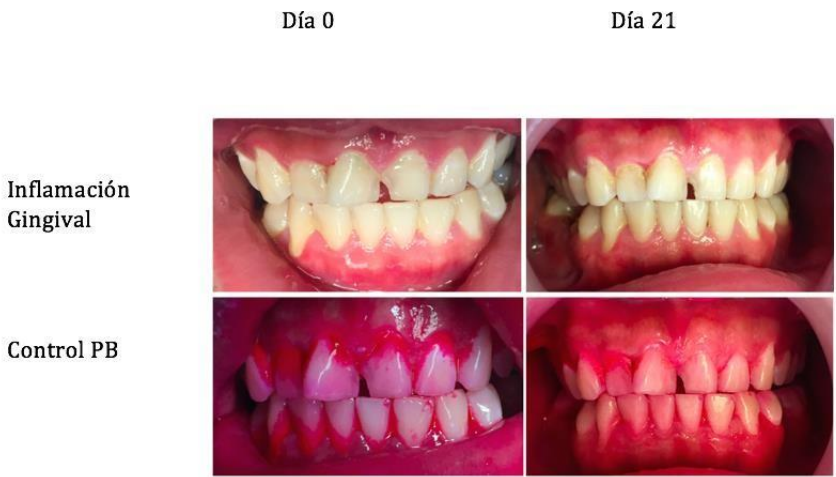

uso como enjuague bucal.

En un régimen de higiene bucal, con la motivación y adiestramiento sobre cepillado dental adecuado, sumadas al uso de un enjuague bucal con hipoclorito de sodio al $0,05 \%$ por 30 segundos dos veces al día, se lograría tener el efecto anti placa y anti gingivitis deseado. Si bien el sabor es fuerte, la alternativa puede ser la utilización del enjuague en una concentración al $0,25 \%$ o $0,20 \%$ por 30

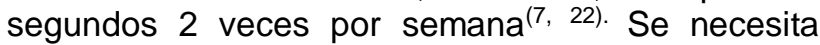
mayor investigación para saber si la mayor frecuencia a menor concentración produce igual efecto que una menor concentración 3 veces al día.
Se concluye que el enjuague bucal de $\mathrm{NaOCl} 0,05 \%$ tiene una actividad comparable al enjuague de CLX $0,12 \%$ usado durante 60 segundos dos veces al día. La CLX $0,12 \%$ mostró resultados antiplaca estadísticamente significativos ( $p=0,001$ ).

El cloro diluido representa una alternativa de bajo costo para lograr efectos similares a la CLX $0,12 \%$ en disminución de inflamación gingival y sangrado al sondaje.

Este estudio presentó limitaciones como la falta de pruebas microbiológicas, el tamaño de la muestra, la disponibilidad total de los sujetos en estudio para las pruebas visuales y el abandono de los pacientes por condiciones sistémicas.

Se sugiere realizar estudios con una muestra más grande y pruebas microbiológicas adecuadas para lograr una mayor validez externa.

Contribuciones de autoría: CEA, JRCA EVC participaron en el diseño del estudio, recolección de datos y análisis de resultados. Todos los autores aprobaron la versión final del artículo.
Fuente de financiamiento: autofinanciado.

Conflictos de interés: Los autores declaran no tener conflicto de interés con el trabajo presentado.

\section{REFERENCIAS:}

1. De Nardo, R. Chiaooel, V. Gomez, M, Romanelli H, Slots, J.Effects of $0.05 \%$ sodium hypochlorite oral rinse on supragingival biofilm and gingival inflammation. Int Dent J 2012; 62: 208-212.

2. Botero JE, Rösing CK, Duque $A$, Jaramillo $A$, Contreras A. Periodontal disease in children and adolescents of Latin America. Periodontol 2000. 2015 Feb; 67(1): 34-57.

3. Morris AJ, Steele J, White DA. The oral cleanliness and periodontal health of UK adults in 1998. Br Dent $\mathrm{J}$ 2002; 191:186-92.

4. Experimental gingivitis in man. II. A longitudinal clinical and bacteriological investigation. Journal of periodontal research. 1966; 1:1-13.

5. Heasman PA, Vernazza CR, Gaunt FL, Pennington MW, Cost-effectiveness of adjunctive antimicrobials in the treatment of periodontitis. Periodontol 2000. 2011 Feb; 55(1): 217-30. 
6. Jorgensen MG, Aalam A, Slots J. Periodontal antimicrobials - finding the right solutions. Int Dent $\mathrm{J}$ 2005 55: 3-12.

7. Zehnder M. Root canal irrigants. J Endod 2006 32: 389-398.

8. Löe H, Silness J. Periodontal disease in pregnancy. I. Prevalence and severity. Acta Odontol Scand 1963 21: 533-551.

9. Turesky S, Gilmore ND, Glickman I. Reduced plaque formation by the chloromethyl analogue of Victamine C. J Periodontol.1970 Jan; 41(1): 41-3.

10. Barnett ML. The role of therapeutic antimicrobial mouthrinses in clinical practice. Control of supragingival plaque and gingivitis. J Am Dent Assoc. 2003 Jun; 134(6): 699-704.

11. Lobene RR, Soparkar PM, Hein JW et al. A study of the effects of antiseptic agents and a pulsating irrigating device on plaque and gingivitis. J Periodontol 1972 43: 564-568.

12. Karpinski TM1, Szkaradkiewicz AK. Chlorhexidine pharmaco-biological activity and application. Eur Rev Med Pharmacol Sci. 2015 Apr; 19(7):1321-6.

13. American Dental Association. Accepted Dental Therapeutics. Chicago, IL: ADA; 1984. p. 326.

14. Jorgensen $M$, Slots J. Antimicrobials in Periodontal Maintenance. Journal of Dental Hygiene, 2001 75: 233-239.

15. Slots J. Low-cost periodontal therapy. Periodontol 2000. 2012 Oct; 60(1):110-37.

16. Slots J. Selection of antimicrobial agents in periodontal therapy. J Periodontal Res 2002: 37: 389398.

17. Slots J, Chen C. The oral microflora and human periodontal disease. In: Tannock GW ed. Medical Importance of the Normal Microflora. London: Kluwer Academic Publishers, 1999: 101-127.

18. Slots J. Anti-infective agents in periodontal treatment. Expert commentary in Medscape Dentistry and Oral Health. September 15, 2011. [Citado: 23 de noviembre de 2016]. Disponible en: http://www.medscape.com/viewarticle/749509.

19. Slots J. The microflora of black stain on human primary teeth. Scand J Dent Res 1974 82: 484-490.

20. Watts A, Addy M. Tooth discolouration and staining: a review of the literature. Br Dent J 2001: 190: 309316.

21. Rich SK, Slots J. Review Sodium hypochlorite (dilute chlorine bleach) oral rinse in patient self-care. J West Soc Periodontol Periodontal Abstr. 2015; 63(4): 99104.

22. Morris AJ, Steele J, White DA. The oral cleanliness and periodontal health of UK adults in 1998. Br Dent $\mathrm{J}$ 2002;191:186-92. 